\title{
Nucleon structure effect on the longitudinal response function
}

\author{
K. Saito ${ }^{a}$ \\ aTohoku College of Pharmacy, Sendai 981-8558, Japan
}

Using the quark-meson coupling model, we study the longitudinal response function for quasielastic electron scattering from nuclear matter within relativistic RPA. In QMC the coupling constant between the scalar meson and the nucleon is expected to decrease with increasing nuclear density. Furthermore, since the electromagnetic form factors of the in-medium nucleon are modified at the same time, the longitudinal response function and the Coulomb sum are reduced by a total of about $20 \%$ in comparison with the Hartree contribution.

\section{Introduction}

There is still considerable interest in the longitudinal response (LR) for quasielastic electron scattering. Within the framework of nonrelativistic nuclear models and the impulse approximation, it is very difficult to reproduce the observed, quenched LR [四]. In the mid '80s, several groups calculated the LR function using the classic version of Quantum Hadrodynamics (QHD-I) [2]. It was argued that the contribution of the relativistic random phase approximation (RRPA), which includes vacuum polarization, is very important in reducing the LR [3].

However, the nucleon has internal structure, and it is nowadays expected that this structure should be modified in a nuclear environment. In QHD-I [2] nuclear matter consists of point-like nucleons interacting through the exchange of scalar $(\sigma)$ and vector $(\omega)$ mesons. (More recent versions of QHD [4 are capable of incorporating the effects of hadron internal structure.) Recently, we have developed a relativistic quark model for nuclear matter, namely, the quark-meson coupling (QMC) model [0], which could be viewed as an extension of QHD-I. However, in QMC the mesons couple to confined quarks and the nucleon is described by the MIT bag model. Using QMC we here report the effect of in-medium changes in the structure of the nucleon on the LR of nuclear matter [6].

\section{Relativistic RPA}

We first review the calculation of the LR function for quasielastic electron scattering from (iso-symmetric) nuclear matter in QHD-I. The starting point is the lowest order polarization insertion (PI), $\Pi_{\mu \nu}$, for the $\omega$ meson. This describes the coupling of a virtual vector meson or photon to a particle-hole or nucleon-antinucleon excitation: $\Pi_{\mu \nu}(q)=$ $-i g_{v}^{2} \int \frac{d^{4} k}{(2 \pi)^{4}} \operatorname{Tr}\left[G(k) \gamma_{\mu} G(k+q) \gamma_{\nu}\right]$, where $G(k)$ is the self-consistent nucleon propagator in relativistic Hartree approximation (RHA) [2]. We can separate the PI into two pieces: 
one is the density dependent part, $\Pi_{\mu \nu}^{D}$, and the other is the vacuum PI, $\Pi_{\mu \nu}^{F}$. The former is finite, but the latter is divergent and must be renormalized [6].

In the Hartree approximation, where only the lowest one nucleon ring is considered, the LR function, $S_{L}^{H}$, is simply proportional to $S_{L}^{H}(q) \propto G_{p E}^{2}(q) \Im \Pi_{L}(q)$. Here $\Pi_{L}\left(=\Pi_{33}-\Pi_{00}\right)$ is the longitudinal (L) component of the PI (we choose the direction of $\vec{q}$ as the $z$-axis) and $G_{p E}$ is the proton electric form factor (EFF), which is usually parametrized by a dipole form in free space: $G_{p E}\left(Q^{2}\right)=1 /\left(1+Q^{2} / 0.71\right)^{2}$ with the space-like momentum transfer, $Q^{2}=-q_{\mu}^{2}$, in units of $\mathrm{GeV}^{2}$.

In RRPA the $\mathrm{L}$ component of the PI, $\Pi_{L}^{R P A}$, involves the sum of the ring diagrams to all orders. It involves $\sigma$ - $\omega$ mixing in the nuclear medium, and is given by $\Pi_{L}^{R P A}(q)=$ $\left[\left(1-\Delta_{0} \Pi_{s}\right) \Pi_{L}+\Delta_{0} \Pi_{m}^{2}\right] / \epsilon_{L}$, where $\epsilon_{L}$ is the L dielectric function [6,7] and $\Delta_{0}$ is the free $\sigma$-meson propagator. Here $\Pi_{s}$ and $\Pi_{m}$ are respectively the scalar and the time component of the mixed PIs. The vacuum component of the scalar PI is again divergent and we need to renormalize it [6, [7]. For the mixed PI there is no vacuum polarization and it vanishes at zero density.

\section{Nucleon structure effects}

To discuss the effect of changes in the internal structure of the nucleon, we consider the following modifications to QHD-I:

1. Meson-nucleon $(\mathrm{N})$ vertex form factor

Since both the mesons and nucleons are composite they have finite size. As the simplest example, we take a monopole form factor, $F_{N}\left(Q^{2}\right)$, at the vertex with a cut off parameter, $\Lambda_{N}=1.5 \mathrm{GeV}$ [6].

2. Modification of the proton electric form factor

We have studied the electromagnetic form factors of the nucleon in nuclear medium, using the QMC model [8]. The main result of that calculation is that the ratio of the EFF of the proton in medium to that in free space decreases essentially linearly as a function of $Q^{2}$, and that it is accurately parametrized as $R_{p E}\left(\rho_{0}, Q^{2}\right) \equiv$ $G_{p E}\left(\rho_{0}, Q^{2}\right) / G_{p E}\left(Q^{2}\right) \simeq 1-0.26 \times Q^{2}$ at $\rho_{B}=\rho_{0}$ (= the normal nuclear matter density) 6, 8 .

3. Density dependence of the coupling constants

In QMC the confined quark in the nucleon couples to the $\sigma$ field which gives rise to an attractive force. As a result the coupling between the $\sigma$ and nucleon is expected to be reduced at finite density [5]. The coupling between the vector meson and nucleon remains constant because it is related to the baryon number.

To study the LR of nuclear matter, we first have to solve the nuclear ground state within RHA [6]. To take into account the modifications 1 and 3 , we replace the $\sigma$ - and $\omega$-N coupling constants in QHD-I by: $g_{s} \rightarrow g_{s}\left(\rho_{B}\right) \times F_{N}\left(Q^{2}\right)$ and $g_{v} \rightarrow g_{v} \times F_{N}\left(Q^{2}\right)$, where the density dependence of $g_{s}\left(\rho_{B}\right)$ is given by solving the nuclear matter problem self-consistently in QMC [6]. Requiring the usual saturation condition for nuclear matter [5], we found the coupling constants: $g_{s}^{2}(0)=61.85$ and $g_{v}^{2}=62.61$ (notice that $g_{s}$ decreased by about $9 \%$ at $\rho_{0}$ ). In the calculation we fix the quark mass, $m_{q}$, to be 5 or 
$300 \mathrm{MeV}, m_{\sigma}=550 \mathrm{MeV}$ and $m_{\omega}=783 \mathrm{MeV}$, while the bag parameters are chosen so as to reproduce the free nucleon mass with the bag radius $R_{0}=0.8 \mathrm{fm}$ [5]. This yields the effective nucleon mass $M^{*} / M=0.81$ at $\rho_{0}$ and the incompressibility $K=281 \mathrm{MeV}$.

\section{Numerical results}

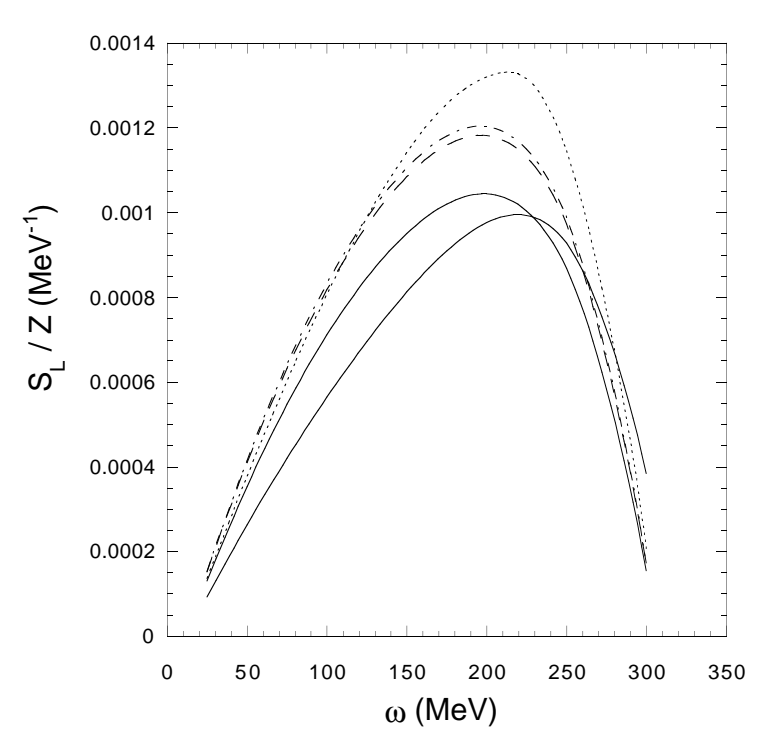

Figure 1. LR functions in QMC. We fix $q=$ $550 \mathrm{MeV}$ and $\rho_{B}=\rho_{0}$. See text for details.

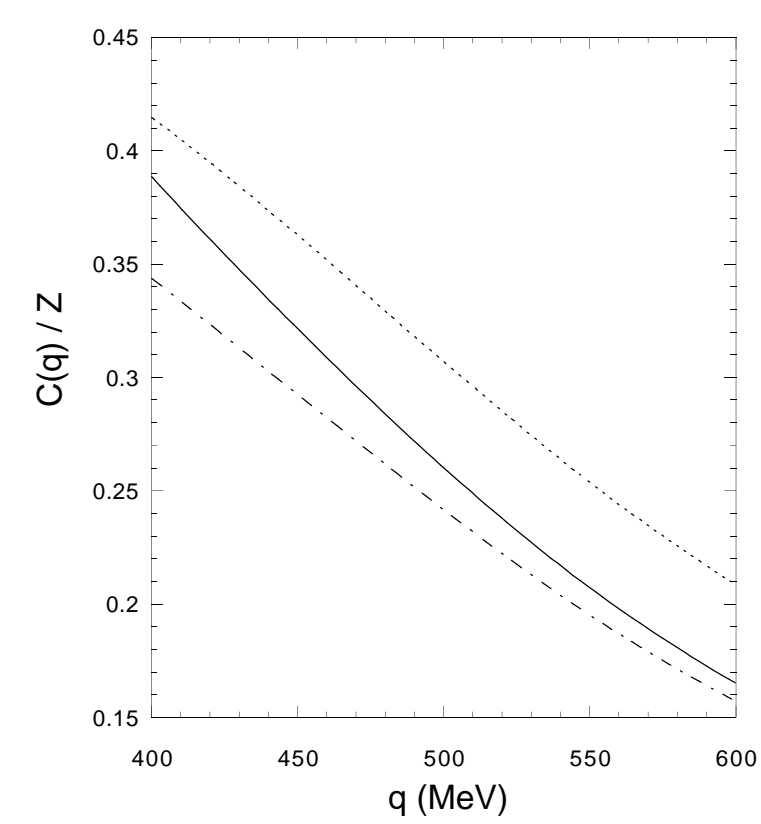

Figure 2. Coulomb sum, $C(q) / Z$, at $\rho_{0}$. See text for details.

Our result is shown in Fig. 11. In the figure, the dotted curve is the result of the Hartree approximation, where the proton EFF is the same as in free space. The dashed curve is the result of the full RRPA, without the modifications 1 and 2 . The dot-dashed curve shows the result of the full RRPA with the meson-N form factor but $R_{p E}=1$. The upper (lower) solid curve shows the result of the full RRPA for $m_{q}=5(300) \mathrm{MeV}$, including all modifications. Because of the density dependent coupling, the reduction of the response function from the Hartree result, caused by the full RRPA, becomes much smaller than that in QHD-I. On the other hand, the modification of the proton EFF is very significant, yielding a much bigger reduction in the response. We can see that the effect of the meson- $\mathrm{N}$ form factor is relatively minor.

It is also interesting to see the quark mass dependence of the LR. As an example, we consider the case of $m_{q}=300 \mathrm{MeV}$. In comparison with the case $m_{q}=5 \mathrm{MeV}$, it is a little smaller and the peak position is shifted to the higher energy transfer side. This is related to the smaller effective nucleon mass in the case $m_{q}=300 \mathrm{MeV}$ than when $m_{q}=$ $5 \mathrm{MeV}$. 
The Coulomb sum, $C(q)=\int_{0}^{q} d q_{0} S_{L}\left(q, q_{0}\right)$, is shown in Fig. 2 as a function of threemomentum transfer, $q$. For high $q$, the strength is about $20 \%$ lower in the full calculation (the solid and dot-dashed curves are for $m_{q}=5$ and $300 \mathrm{MeV}$, respectively) than for the Hartree response with $R_{p E}=1$ (the dotted curve). For low $q$, the full calculation with the constituent quark mass remains much lower than the Hartree result, while in case of the light quark mass it gradually approaches the Hartree one. This difference is caused by that the effective nucleon mass for $m_{q}=5 \mathrm{MeV}$ being larger in matter than that for $m_{q}=300 \mathrm{MeV}$.

We comment on the transverse response (TR) from nuclear matter. In Ref. [8] it was found that the in-medium modification of the nucleon magnetic form factor within QMC is very small. Therefore, one would expect the total change in the TR caused by RRPA correlations and the effect of the variation of the structure of the nucleon to be much smaller than in the LR.

\section{Summary}

We have calculated the LR of nuclear matter using the QMC model. The reduction of the $\sigma$-N coupling constant with density decreases the contribution of the RRPA, while the modification of the proton EFF in medium reduces the LR considerably. The LR or the Coulomb sum is reduced by about $20 \%$ in total, with RRPA correlations and the variation of the in-medium nucleon structure contributing about fifty-fifty. It will be interesting to extend this work to calculate both the LR and TR functions for finite nuclei, in order to compare directly with the new experimental results which are anticipated soon [9].

The author would like to thank A.W. Thomas, K. Tsushima, M. Ericson, P.A.M. Guichon, W. Bentz and J. Morgenstern for valuable discussions. This work was supported by the Japan Society for the Promotion of Science.

\section{REFERENCES}

1. Z.E. Meziani et al., Phys. Rev. Lett. 52 (1984) 2130; W.M. Alberico, P. Czerski, M. Ericson and A. Molinari, Nucl. Phys. A 462 (1987) 269.

2. B.D. Serot and J.D. Walecka, Adv. Nucl. Phys. 16 (1986) 1.

3. For example, H. Kurasawa and T. Suzuki, Nucl. Phys. A 445 (1985) 685; C.J. Horowitz, Phys. Lett. B 208 (1988) 8.

4. B.D. Serot and J.D. Walecka, Int. J. Mod. Phys. E 6 (1997) 515.

5. P.A.M. Guichon, K. Saito, E. Rodionov and A.W. Thomas, Nucl. Phys. A 601 (1996) 349; K. Saito, K. Tsushima and A.W. Thomas, Nucl. Phys. A 609 (1996) 339; Phys. Rev. C 55 (1997) 2637; K. Saito, nucl-th/9811088.

6. K. Saito, K. Tsushima and A.W. Thomas, Phys. Lett. B 465 (1999) 27.

7. K. Saito, K. Tsushima, A.W. Thomas and A.G. Williams, Phys. Lett. B 433 (1998) 243.

8. D.H. Lu, A.W. Thomas, K. Tsushima, A.G. Williams and K. Saito, Phys. Lett. B 417 (1998) 217; D.H. Lu, K. Tsushima, A.W. Thomas, A.G. Williams and K. Saito, Phys. Rev. C 60 (1999) 068201.

9. J. Morgenstern, private communication. 